REFLEXIÓN

Recibido: $15 / 10 / 2013$

Revisado: 15/11/2013

Aprobado: 15/12/2013

\title{
EL DESARROLLO LOCAL. PROPUESTAS Y CONDICIONES NECESARIAS
}

\author{
RICARDO RAÚL GUTIÉRREZ \\ Departamento de Economía. \\ Universidad Nacional del Sur. Bahía Blanca. Argentina.
}

\section{RESUMEN}

El desarrollo local ha estado adquiriendo importancia en el mundo, como opción a la extensión de la globalización que se inicia en la década del 70. Como la problemática de la globalización excede el ámbito económico, ocurre lo mismo con el desarrollo local.

La apertura de las economías, el incremento de la productividad y la pérdida de las consiguientes protecciones, obligaron a reestructurar los sistemas productivos y las producciones. La propuesta de política de desarrollo local proyecta experiencias nacionales en países desarrollados, que consiguieron enfrentar, más o menos exitosamente, los problemas que debieron enfrentar cuando la competencia de otros países destruyó parte de sus estructuras productivas. La aplicación de políticas inspiradas en el desarrollo local se plantea como alternativa a desarrollar. En el discurso político se han popularizado varios de los instrumentos que estas políticas promueven, tales como la colaboración público-privada, las incubadoras, la transferencia de tecnología, la acción educativa y de investigación, la asociatividad entre actores, etc. La aplicación de estas medidas no ha sido todo lo exitoso que puede serlo, dadas las experiencias en la práctica de estas políticas en diversos países del mundo. Se analiza esta diferencia de resultados a partir de diferencias en las condiciones iniciales e inexistencia de situación de confiabilidad política, escasez de capital social y de cooperación, como asimismo errores de política pública.

Palabras clave: Desarrollo Local. Globalización. Política Económica. Estrategias. Pymes.

\section{ABSTRACT}

Local development has been gaining importance in the world, as an option to the spread of globalization that began in the 1970s. As the problem of globalization exceeds the economic sphere, the same is true of local development.

The opening of economies, the increase of productivity and the loss of the consequent protections, forced to restructure the productive systems and the productions. The proposed local development policy projects national experiences in developed countries that were able to face, more or less successfully, the problems they faced 
when competition from other countries destroyed some of their productive structures. The implementation of policies inspired by local development is proposed as an alternative to develop. In the political discourse, several of the instruments that these policies promote have been popularized, such as public-private collaboration, incubators, technology transfer, educational and research action, associativity among actors, etc. The implementation of these measures has not been all that successful, given the experiences in the practice of these policies in various countries of the world. This difference in results is analyzed based on differences in the initial conditions and lack of a situation of political reliability, lack of social capital and cooperation, as well as errors of public policy.

Key words: Local Development. Globalization. Economic Policy. Strategies. SMEs.

\section{Introducción}

A partir de un diagnóstico panorámico de los efectos de la globalización y sus efectos en los ámbitos locales territoriales, se proponen una cierta cantidad de medidas englobadas en la denominada corriente de desarrollo local. Se analizan las particularidades generales de esta propuesta y se concluye sobre la factibilidad y posibilidad de ponerla en vigencia, total o parcialmente, con posibilidades de obtener éxito en el desarrollo, entendido como concepción de definición múltiple, con fuerte énfasis en lo económico, pero con componentes políticos y sociales imprescindibles.

\section{La situación en el ámbito local territorial}

La globalización afecta a todos los sectores de una región. Sin embargo, la intensidad y la forma en que lo hace, manifiesta diferencias importantes. En primer lugar, en razón de la ineludible relevancia del territorio y sus características ecológicas y ambientales para la producción de bienes y las especificidades especiales y particulares de la mano de obra.

En segundo lugar, el impacto es diferente en el sector agropecuario res- pecto al sector industrial. El primero produce fundamentalmente alimentos e insumos básicos, y por ello la cadena de producción y el consumo de los mismos son particulares. La disponibilidad y el acceso a los productos alimenticios determinan los niveles de satisfacción o hambre de los pueblos, una cuestión particularmente sensible. Correlativamente, el sector industrial produce bienes indicativos, pero esencialmente otorga empleo en mejores condiciones de trabajo. Estos efectos no son meramente económicos, ya que también se expresan en términos de la gobernabilidad ante carencias alimenticias y el desempleo. En tercer lugar, la demanda global mundial, sobre todo la potencial, de alimentos y de empleo es superior a la oferta. Finalmente, los desenvolvimientos del agro y la industria establecen, en gran medida, el marco y las limitaciones del desarrollo de los sectores de servicios. Estos efectos dependen del cambio tecnológico, pero son perceptibles fácilmente. Estos nuevos escenarios plantean situaciones y problemas novedosos. Obviamente, un aumento en la demanda mundial provoca un incremento de precios y la globalización facilita la difusión de ello, lo que favorece a las regiones productoras, pero perjudica a los consumidores. 
El estímulo para ampliación de la producción afecta a toda la actividad productiva, alguna positivamente (mayor demanda de bienes) o negativamente (demandas de bienes que sustituyen producciones existentes). Para incrementar y reorientar la producción se requieren cambios tecnológicos, incremento de la densidad de capital y capacitación de la mano de obra. Las consideraciones ecológicas y los aspectos culturales e institucionales, determinan fuertemente el comportamiento del sector productivo ante las nuevas realidades. Esto es así porque, en mayor medida cuando existe un alto grado de heterogeneidad, los cambios son tanto de capacidades como de decisiones que se toman individual y colectivamente; pero que esencialmente dependen del productor, de su cultura, de sus valores, de sus ambiciones económicas. La mera existencia de oportunidades de producción y de innovación tecnológica a partir de tecnologías existentes, no significa la materialización de decisiones positivas en el sentido productivo.

En este punto, la problemática que enfrentan las empresas medianas y pequeñas y los productores individuales es como conseguir eficiencia productiva y permanecer en la producción. El aumento de precios resultante de los aumentos de demandas, viene acompañado de requerimientos de calidad y de especificaciones que dependen mayoritariamente de nuevas tecnologías duras y blandas que significan costos incrementales. Las grandes empresas consiguen captar más fácilmente el diferencial de beneficios producido por estos cambios, en razón de sus economías de tamaño y su mejor conocimiento de las instituciones vigentes, del funciona- miento de los mercados, de los proveedores de insumos y de los clientes en la cadena productiva; y seguramente también de su capacidad de negociación con las autoridades políticas y con todo el sistema productivo (financiero, servicios, etc.). El resultado suele ser el desplazamiento de las empresas y productores menores en tamaño, lo que territorialmente suele provocar sustitución productiva de ellos por las grandes empresas, lo que a corto plazo deviene en desempleo, lo que se transforma en un problema político regional, y a veces nacional. La cuestión de las políticas públicas resulta complejo de analizar, en razón de que los objetivos de diferentes niveles o agencia de gobierno son, a menudo, notoriamente diferentes e incluso contradictorios. En general, se ha observado una reacción de los gobiernos nacionales, de deprimir el efecto interno del aumento internacional de precios, sobre todo en los bienes alimenticios, dada su incidencia en el costo de vida, fundamentalmente de los habitantes urbanos, o por la intencionalidad fiscal de apropiarse de los posibles (y reales) incrementos de la rentabilidad de la actividad para financiar políticas y estructura del estado. Además los gobiernos, actúan en términos de sus compromisos de sustentación política, de los grupos de interés que participan de la actividad y la acción gubernamental, de su conocimiento y percepción de la complejidad de la situación interna e internacional, y de los cambios esperables o supuestos. En resumen, puede expresarse que los efectos de la globalización y de la aparición de nuevas tecnologías de producción, pueden estar disponibles en cualquier lugar del mundo, tanto como presiones para aumentos de la pro- 
ducción como para desplazamiento y sustitución de agentes productivos. La reacción interna depende del comportamiento tanto de los agentes productivos nacionales como de las políticas públicas y de los objetivos y la eficiencia de la acción operativa de los gobiernos. Resulta redundante explicitar que estas acciones, pueden ser parte de la agenda política de los gobiernos, y ser manifestación de problemáticas internas, independientemente de los procesos mundiales. En estas circunstancias, se generará una presión adicional por los efectos indicados de la ocurrencia de la globalización, en el marco del cambio tecnológico, de un valor positivo en el desarrollo económico mundial y de un crecimiento de la población. Los problemas emergentes no se agotan en el ámbito interno de los países, porque la propia dinámica y presiones de ampliación de la producción, en numerosos casos, termina presionando sobre las relaciones internacionales, cuando se proponen proyectos multinacionales y políticas que trascienden las fronteras, impulsadas por países, por grupos o por sectores de poder internacional y por organismos supranacionales.

Las políticas de desarrollo local y territorial. Las denominadas políticas de desarrollo local, identificadas con esta denominación, se pergeñaron y aplicaron esencialmente en el ámbito productivo europeo, en sus distintas manifestaciones. Algunos autores, partiendo de una concepción que enfatiza en mayor medida nuevas líneas de la teoría del desarrollo, impulsaron las políticas de desarrollo endógeno.

En parte fueron motivadas por las necesidades de adecuación de los países que, después de la segunda guerra mundial, dieron los primeros pasos de la actual Comunidad Europea, originariamente fundada en el ámbito de lo económico. A partir de las políticas globales, desde mediados de los $70 \mathrm{y}$ de la ampliación de la experiencia europea de integración, se aplicaron en un sentido más extendido y perentorio, derivado de los compromisos de reducción del proteccionismo de los países desarrollados. Por ello, existían las necesidades de defender las actividades económicas ya existentes y en inducir nuevos emprendimientos, como política para solucionar problemas de competitividad respecto de las grandes empresas transnacionales, algunas de los propios países europeos y otras de carácter internacional. La dinámica europea en esta experiencia, amplió la conceptualización para llevarla también al ámbito de lo político y social, inducido por la necesidad de asegurar la gobernabilidad en contextos de necesarias reestructuraciones y ajustes derivadas de la redefinición de los roles en esta nueva estructura mundial. Como esto implicaba encontrar sustitutos para actividades que, se realizaban en espacios territoriales propios que sufrían los perjuicios de la transformación, se avanzó en el análisis espacial y se amplió la denominación de la política con este nuevo calificativo. Ello resultó natural por cuanto se hizo evidente que la problemática afectaba directamente situaciones regionales: infraestructura, educación, logística; pero también decisiones políticas sobre desconcentración del poder, ecología, identidad, etc.

En realidad se avanzó en una concepción que había quedado subsumida en las últimas centurias por el desarrollo del estado nación y la centralidad 
del poder en muchos países europeos, pero que no necesariamente era nueva. Asimismo, también pueden buscarse antecedentes paralelos y con ciertas ideas comunes con los mecanismos de emprendimientos, algunos incluso del Medioevo europeo, expresados en forma colaborativa, de autogestión, etc.

También pueden encontrarse ejemplos en todo el mundo. Por su relevancia, resulta particularmente interesante la experiencia en el interior de EE.UU. a partir de los principios del comunitarismo, el diseño institucional productivo de autogestión de la ex Yugoeslavia después de la segunda guerra mundial, experiencias llevadas adelante en distintos lugares de Asia, expresiones del cooperativismo en todo el mundo, etc. En América Latina, experiencias con algunos de estos elementos, pueden encontrarse en la acción de comunidades ligadas por determinados valores que las constituían.

El hecho distintivo es que estas políticas son el resultado de análisis parciales, experiencias históricas económicas y políticas, soluciones puntuales a problemas específicos. Se agruparon en una suerte de propuestas, que en parte se superponen o dejan lugar para incorporaciones idiosincráticas. No existe detrás de estas políticas un esquema global como puede pensarse en la teoría de los mercados en el liberalismo, o de la teoría de la planificación central en el colectivismo. Por ello, existen numerosos trabajos que incorporan distintas herramientas con diferente grado de importancia.

En realidad este problema no inhabilita la aplicabilidad, si se admite que la teorización racional es relativa y el mundo en el que se vive es un mundo complejo, con alta proporción de incertidumbre, con situaciones emergentes imprevistas, que obligan a aceptar políticas fundadas en principios sujetos a revisión permanente. Esto tiene algunos inconvenientes metodológicos por la falta de certidumbre y previsión exacta; pero esta es una situación que también alcanza a la política económica fundada en teorías racionales, que siempre tienen limitaciones de las hipótesis y por la dinámica social en la cual es difícil postular una racionalidad similar a la abstracción de los modelos.

La exposición siguiente de los principios básicos de las políticas de desarrollo social territorial es una conceptualización utilizada en líneas de investigación y con las características particulares del territorio donde se han realizado los análisis, a partir de diagnósticos propios. No se considera que ello constituye una limitación severa al estudio de las propuestas de políticas, pero tiene sus particularidades y por ello, requiere confrontación con otras realidades. Los principios básicos de esta conceptualización son los siguientes $^{1}$ :

1) "Un rol del estado que incluye una fuerte participación del ciudadano en las decisiones, no sólo en términos electorales, con cuotas significativas de transparencia y descentralización (no sólo relocalización de funciones y decisiones).

1. Se reproducen literalmente de Gutiérrez, Ricardo Raúl. La problemática del desarrollo local en el sudoeste bonaerense. En Diez, José y Ricardo Raúl Gutiérrez (2008) a). Capítulo IV. 
2) La generación de actitudes proactivas de los gobiernos locales en términos de institucionalizaciones que privilegien la racionalidad y la eficiencia en el uso de recursos, con el objetivo de lograr procesos de desarrollo exitosos.

3) Elaboración de una estrategia territorial de desarrollo que obtenga el apoyo de las instancias regionales, provinciales y nacionales de los gobiernos y de los agentes individuales y organizaciones privadas; con consensos respecto a las metas básicas y aceptación de diversidad política e ideológica dentro de esos consensos.

4) Amplia coordinación de programas e instrumentos de desarrollo por parte de algún organismo consensuado en su estructura y funcionamiento, absolutamente identificado con los objetivos, y eficiente en su operación.

5) Fomento permanente de empresas locales y capacitación asistida por los recursos humanos existentes.

6) Asociativismo entre todos los agentes sociales, tanto públicos como privados, con consenso sobre los alcances y ámbitos de esta relación. En este espacio, aparece la referencia a la creación de redes sociales que necesariamente han de funcionar en términos de eficiencia y de generación de sinergia y economías externas apropiables por los miembros de la red.

7) Desarrollo de equipos de liderazgo social local que estén en condiciones de explicitar, comunicar y promover la movilización y participación en los procesos de desarrollo de todos los actores locales". Cada una de estas acciones tiene condicionantes según los valores y objetivos que se presentan en cada uno de los agentes intervinientes y según las condiciones de vinculación, que determinan la trama real de convivencia social, económica y política. Con referencia a la participación de los ciudadanos en las decisiones, actividades y roles de los gobiernos, más allá de las cuestiones electorales, se plantean algunas cuestiones que resultan problemáticas. Tiene que verificarse con carácter general, dado que se hace necesario evitar toda sospecha de discriminación, que perturbaría el apoyo de sectores de ciudadanos. La población rural y periurbana, en grado variable según los regiones, se encuentra localizada en forma dispersa, y los medios de comunicación no tienen el alcance que se puede verificar en el ámbito urbano. Obtener éxito en la participación efectiva en decisiones y en forma estructural es complejo.

Probablemente se verifique en forma esporádica e incluso aleatoria, según los tiempos de trabajo. Requiere una predisposición amigable de políticos y administradores, y una metodología de compartir la gestión. Respecto a la realización por parte de los gobiernos locales de actividades para promover e institucionalidad prácticas en búsqueda de racionalidad y eficiencia; puede considerarse que requiere imprescindiblemente la participación de los sectores técnicos y profesionales y del sistema educativo y de investigación. Existe aquí un rol significativo para las escuelas técnicas y para las universidades, pero no resulta simple la 
acción conjunta de actividades con las personas en su carácter de ciudadanos, de empresarios, de trabajadores, de políticos y de administradores públicos; y que no se transformen expresando situaciones de conflictos preexistentes o larvados. Ya la propia convivencia puede ser difícil, pero puede ser peor para determinar conjuntamente la aplicación de prácticas y procedimientos que implican la incorporación de principios de eficiencia y responsabilidad, con sus costos y esfuerzos imprescindibles para algunos. Resulta obvio que los beneficiarios de un proceso exitoso será toda la población, pero el rol de los gobiernos locales es vital para que la ciudadanía y los agentes económicos reconozcan que su gobierno cercano actúa en forma de proteger la actividad local, en términos de generar eficiencia operacional. Además, los gobiernos locales deben evidenciar su intencionalidad de defender la situación local en los demás niveles de gobierno que también deben colaborar positivamente, y manifestarse en ese sentido permanentemente. En general, debe evitarse la percepción popular de un bajo interés de los sectores políticos cuanto más se alejan de lo local, por mayores concentraciones de votantes no locales. En general, puede considerarse que existe, se genera una mayor respuesta política a las demandas de los sectores más urbanizados con mayor capacidad de demanda y presión. Es necesario romper con este concepto, con acciones y realidades, so pena de generar más reacciones de inmigración hacia centros urbanos de desarrollo local territorial. Un problema invalidante, se presenta cuando se perciben situaciones prebendarias. En la percepción ciudadana local, se sustituye el esfuerzo para el desarrollo terri- torial por la búsqueda del subsidio desvinculado con el proceso de desarrollo.

La elaboración de una estrategia territorial de desarrollo requiere de un grado de conocimiento e información tanto de los aspectos económicos como de las instituciones vigentes, y la creación en nuevas. Este proceso resulta imprescindible porque resulta en una expresión que todos pueden consultar y que constituye un marco referencial fáctico que ayuda al convencimiento popular. Algunas de estas instituciones pueden ser locales, pero seguramente muchas de ellas provienen de otros niveles más amplios de gobierno, y el conocimiento de ellas puede ser inadecuado e insuficiente. Aquí se hace imprescindible de nuevo, la participación de los sectores educativos, por cuanto se requiere capacidad técnica para definir estrategias y para expresarlas en documentos, que deben ser idóneos para la información y el consenso de los sectores locales, pero también en niveles nacionales e intermedios de los gobiernos, para lograr compromisos de acción efectiva.

En esta misma inteligencia se identifica la necesidad de la constitución de un organismo consensuado en su estructura y funcionamiento, con el fin de realizar amplia coordinación de programas e instrumentos de desarrollo. La existencia de un organismo que realice la aplicación de la política como actividad central en su existencia resulta imprescindible para identificar el compromiso político; pero también es insoslayable la participación local en ese organismo, con capacidades de participación en las decisiones, y con carácter permanente y efectivo. Particularmente sensible en estas instituciones, resulta el diálogo 
técnico, para evitar la incomprensión y el hermetismo esotéricos. Es necesaria la comunicación y el convencimiento de todas las partes y el reconocimiento de los demás agentes involucrados en la actividad. El ente administrador, debe ser aceptado y sus distintas áreas deben ser consideradas como propias y necesarias por todos, para lograr identificación con los objetivos y el reconocimiento de la eficiencia operacional como valores determinantes de la obtención de resultados exitosos.

En este contexto, la actividad de fomento permanente de empresas locales y de capacitación es un pilar imprescindible. Para el gobierno, significa la necesidad de asignar fondos permanentemente, hasta conseguir resultados de sustentabilidad. Como la política de desarrollo local se plantea en términos de participación, no puede decaer la proactividad pública, para conseguir y mantener la proactividad en los términos del esfuerzo de los ciudadanos y agentes económicos involucrados. El carácter de permanente resulta de los tiempos necesarios de maduración de las políticas y de la formación de las personas para el comportamiento productivo eficiente. Resulta obvio que no se descarta el subsidio, pero este debe tener el objetivo de obtener las condiciones de desarrollo. Asimismo, la actitud de mejoramiento permanente y de incorporación de tecnologías deben ser parte en la dinámica de los organismos y agentes. Aquí también, la participación de instancias y organizaciones vinculadas con la enseñanza y la investigación resultan imprescindibles. Pero fundamentalmente, es necesario que estas actividades se planteen como un proceso sistemático y permanente, y no como un acto que permite ascender un escalón en el desarrollo, pero que se inmoviliza allí. Esto resultaría incongruente con la competencia globalizada, pero también con la efectividad de la política y sus instituciones.

La asociatividad entre todos los agentes sociales debe ser considerada un valor constituyente de la política. Ello implica un reconocimiento de la dificultad del éxito individual. El éxito individual no tiene porque ser considerado como pernicioso, excepto cuando imposibilita o dificulta las posibilidades de mejoramiento y de avance de los demás. La asociatividad se considera en un sentido amplio, que alcance tanto a la iniciativa privada como pública, con el convencimiento de su relevancia y de sus alcances. No sólo alcanza a la creación de redes sociales y de vinculación estructural de sectores privados y públicos, sino a la interacción entre los organismos de los gobiernos locales y de estos con los organismos de otros niveles superiores del gobierno e internacionales.

Un proceso de este tipo necesita imprescindiblemente de liderazgo social local para conseguir resultados exitosos. Pero es un liderazgo especial, que requiere que transmita y manifieste que está dirigido a liderar un proceso de estas características. Debe estar institucionalizado y ser preferiblemente colectivo, para evitar la vulnerabilidad de los desvíos individuales, porque la esencia del proceso es necesariamente compartida. El liderazgo resulta particularmente determinante en las fases iniciales, pero necesita su presencia efectiva y permanente. 


\section{A modo de conclusión}

Resulta fácil de comprender que el cumplimiento de esta cantidad de requerimientos no es fácil de conseguir. Se requiere una maduración política y social que, no necesariamente significa homogeneizar la sociedad, sino en convencer a todos los agentes sociales de asociarse, para actuar racionalmente en beneficio de todos, definiendo objetivos y líneas de conducta que sean percibidos como de beneficio general, y actuar en consecuencia en términos de las decisiones que se tomen y que se apoyen. La identificación de este comportamiento con los bases de la democracia resultan evidentes.

También resulta fácil de comprender que este comportamiento es posible, pero habitualmente requerirá de confianza en los liderazgos y las instituciones, para lo cual la transparencia y participación resulta imprescindible. Es decir, se necesita capital social, con el concepto habitualmente utilizado en la literatura, pero no es suficiente. Las medidas que se instrumenten deben ser posibles, creativas y eficientes; y com- prenderse la relevancia de la acción común en definir, actuar y permanentemente corregir los procesos de desarrollo.

Existen casos exitosos en el mundo, con muchas particularidades. Estas experiencias han necesitado de tiempo y esfuerzo, pero han permitido conseguir niveles de bienestar que, en algunos momentos anteriores, eran inexistentes e imposibles de lograr.

La dificultad de conseguir una trama completa de política, no invalida sus posibilidades parciales. Esta parcialidad puede entenderse, tanto en el alcance territorial, como en la intensidad de aplicación, como en la adhesión de sólo determinados sectores de la actividad social y productiva. Probablemente esta situación parcial resulta más realista en las políticas concretas, y las experiencias existentes manifiestan resultados concretos siendo, en gran medida, de este tipo. Pero la falta de participación e integración total provoca la pérdida de sinergias de definición y operacionales, que suelen ser elementos muy relevantes en este tipo de procesos. 


\section{BIBLIOGRAFÍA}

Diez, José lgnacio y Ricardo Raúl Gutiérrez (2008a). Acción Colectiva, innovación y planificación del desarrollo. Bahía Blanca: Ed. Ediuns.

(2008b). Transformaciones en la gestión municipal y políticas para el desarrollo local. Bahía Blanca: Ed. Ediuns.

Gutiérrez, Ricardo Raúl (2006). La inversión en capital humano y social: relaciones entre gobiernos, universidad y agentes productivos en el desarrollo local. IX Seminario Internacional de la Red Interamericana de Investigadores sobre globalización y territorio. Bahía Blanca: Departamentos de Economía y Geografía y Turismo. 